\title{
Least-squares Multirate FIR Filters
}

Roberto Manduchi

Pietro Perona

Computer Science Department Dept. of Electrical Engineering

\author{
Stanford University California Institute of Technology \\ Stanford, CA $94305 \quad$ Pasadena, CA 91125 \\ Fax $(415) 725.1449 \quad$ Fax $(818) 395.2137$ \\ manduchi@cs.stanford.edu perona@vision.caltech.edu \\ January 30, 1996
}

\begin{abstract}
The authors propose a new least-squares design procedure for multirate FIR filters with any desired shape of the (band-limited) frequency response. The aliasing, inherent in such systems, is implicitly taken into account in the approximation criterion.
\end{abstract}




\section{Introduction}

The multirate implementation of FIR filters (see figure 1), introduced by Rabiner and Crochiere [1], makes for reduced computational complexity. In fact, the samples at the output of the FIR filter $g(n)$ that are deleted by the $M$-fold sampler do not need to be computed, and the null-valued samples introduced by the $M$-fold interpolator do not contribute to the convolution operated by the FIR filter $h(n)$. Only the case of brick-wall frequency response was considered in [1], and the design technique was inspired by minimax criteria.

We propose a least-squares criterion for the design of multirate FIR filters, to approximate the spectral shape of any desired prototype $d(n)$ (assuming that the necessary bandlimiting conditions are met, i.e. that the spectral support of $d(n)$ has length less than $2 \pi / M)$. The resulting system is linear periodically time-invariant (LPTV [2]), and it is characterized by the $M$ impulse responses $\left\{t^{(i)}(n+i), 0 \leq i<M\right\}$, corresponding to the $M$ inputs $\{\delta(n+i), 0 \leq i<M\}$. The fact that the impulse responses differ from each other is usually referred to as aliasing effect. The least-squares criterion introduced in this Letter makes for the joint reduction of the approximation error and of the inherent aliasing. 


\section{Theory}

We consider here only the case $M=2$ (definitions and results are extended straightforwardly to the case of higher $M)$. Define the polyphase components [2] of $g(n)$ as

$$
g_{0}(n)=g(2 n), g_{1}(n)=g(2 n+1)
$$

One easily shows [2] that

$$
t^{(0)}(n)=h * \bar{g}_{0}(n), t^{(1)}(n+1)=h * \bar{g}_{1}(n)
$$

where $\bar{g}_{0}(n)$ and $\bar{g}_{1}(n)$ are obtained by interleaving $g_{0}(n)$ and $g_{1}(n)$ with null-valued samples.

We propose the following design criterion: given the kernel $d(n)$ to be approximated, find the filters $g(n)$ and $h(n)$ with given length $N_{g}$ and $N_{h}$ respectively, which minimize the approximation error $\mathcal{E}^{2}$, defined as

$$
\mathcal{E}^{2}=\frac{\left\|t^{(0)}(n)-d(n)\right\|^{2}+\left\|t^{(1)}(n)-d(n)\right\|^{2}}{2}
$$

Term $\mathcal{E}^{2}$ implicitly accounts for both the approximation quality and the aliasing. In fact,

if $\mathcal{E}^{2}$ is small, we may expect both the system's impulse responses to be "close" to $d(n)$, and 
therefore "close" to each other. More precisely, the following upper bound holds:

$$
\left\|t^{(0)}(n)-t^{(1)}(n)\right\|^{2} \leq 2\left(\mathcal{E}^{2}+\left\|t^{(0)}(n)-d(n)\right\|\left\|t^{(1)}(n)-d(n)\right\|\right)
$$

No simple closed form solution can be found to the minimization problem, since the error $\mathcal{E}^{2}$ in (1) is composed of quadratic forms of bilinear expressions in $g(n)$ and $h(n)$. A standard procedure in such cases is based on iterative minimization [3]. Our iterative algorithm is briefly outlined in the remainder. Vectorial notation is used for sequences: a sequence $x(n)$ is represented by a column vector $\mathrm{x}$ whose entries are the samples of $x(n)$. Symbol "T" stands for vector/matrix transposition. We start from an initial guess of $g_{0}(n)$ and $g_{1}(n)$, and then iterate through the following two steps:

Optimization of $h(n)$ for fixed $g_{0}(n), g_{1}(n)$.

Let $\overline{\mathbf{G}}_{0}$ and $\overline{\mathbf{G}}_{1}$ be the Toeplitz matrices representing the filtering with $\bar{g}_{0}(n)$ and $\bar{g}_{1}(n)$ respectively. Then

$$
\mathcal{E}^{2}=\frac{\left(\overline{\mathbf{G}}_{0} \mathbf{h}-\mathbf{d}\right)^{T}\left(\overline{\mathbf{G}}_{0} \mathbf{h}-\mathbf{d}\right)+\left(\overline{\mathbf{G}}_{1} \mathbf{h}-\mathbf{d}_{+}\right)^{T}\left(\overline{\mathbf{G}}_{1} \mathbf{h}-\mathbf{d}_{+}\right)}{2}
$$

where $\mathbf{d}_{+}$is the vector representing $d(n+1)$. Hence, $\mathcal{E}^{2}$ is minimized for

$$
\mathbf{h}=\left(\overline{\mathbf{G}}_{0}^{T} \overline{\mathbf{G}}_{0}+\overline{\mathbf{G}}_{1}^{T} \overline{\mathbf{G}}_{1}\right)^{-1}\left(\overline{\mathbf{G}}_{0}^{T} \mathbf{d}+\overline{\mathbf{G}}_{1}^{T} \mathbf{d}_{+}\right)
$$

Optimization of $g_{0}(n)$ and $g_{1}(n)$ for fixed $h(n)$. 
Let $\mathbf{H}$ be the Toeplitz matrix representing the convolution with $h(n)$, and let $\mathbf{U}$ be a matrix obtained by interleaving the rows of a suitably sized identity matrix with null-valued rows. Then

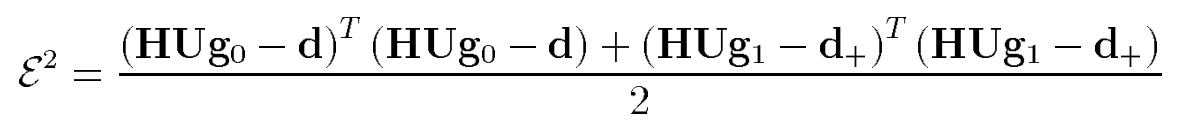

Error $\mathcal{E}^{2}$ is minimized for

$$
\mathbf{g}_{0}=\left(\mathbf{U}^{T} \mathbf{H}^{T} \mathbf{H U}\right)^{-1} \mathbf{U}^{T} \mathbf{H}^{T} \mathbf{d}, \mathbf{g}_{1}=\left(\mathbf{U}^{T} \mathbf{H}^{T} \mathbf{H} \mathbf{U}\right)^{-1} \mathbf{U}^{T} \mathbf{H}^{T} \mathbf{d}_{+}
$$

Since the error $\mathcal{E}^{2}$ does not increase at any iteration and is bounded from below by zero, we are guaranteed to converge to some minimum of $\mathcal{E}^{2}$. However, the minimum may be just local, and it may be useful to run the algorithm several times with different starting points, choosing the solution that gives the smallest $\mathcal{E}^{2}$.

\section{A design example}

We have tested the proposed design technique for a kernel $d(n)$ shaped as the second derivative of a gaussian function, a filter widely used in computer vision (see figure 2). The standard deviation $\sigma$ was set to 10 and the length of $d(n)$ was 47 samples. The design parameters were: $M=4, N_{g}=N_{h}=25$. The multirate implementation thus requires approximately four times fewer elementary operations per input sample than the direct implementation of $d(n)$. The starting point for the iterative optimization was a constant sequence $g(n)$. 
In order to evaluate the multirate system's performance, we may define the signal to approximation noise ratio:

$$
S N R=\frac{\|d(n)\|^{2}}{\mathcal{E}^{2}}
$$

and the signal to aliasing ratio:

$$
S A R=\frac{\|d(n)\|^{2}}{\max _{i, j}\left\{\left\|t^{(i)}(n)-t^{(j)}(n)\right\|^{2}\right\}}
$$

In our case, we obtained $S N R=28.3 \mathrm{~dB}$ and $S A R=25.8 \mathrm{~dB}$.

\section{Conclusion}

The multirate implementation of band-limited FIR filters makes for the reduction of the computational weight. We have presented a novel least-squares technique to design multirate FIR filters for any shape of the (band-limited) desired frequency response. The technique is based on temporal domain approximation, and the error criterion accounts for both goodness of approximation and aliasing.

\section{References}

[1] RABINER, L.R. and CROCHIERE, R.E.: 'A novel implementation for narrow-band FIR filters', IEEE Trans., October 1975, ASSP-23, pp.457-464. 
[2] VAIDYANATHAN, P.P.: 'Multirate systems and filter banks' (Prentice Hall, Englewood Cliffs, NJ, USA, 1993).

[3] GURSKI, G.C., ORCHARD, M.T. and HULL, A.W.: 'Optimal linear filter for pyramidal decomposition', Proc. IEEE ICASSP'92, 1992, San Francisco, pp. 633-636. 


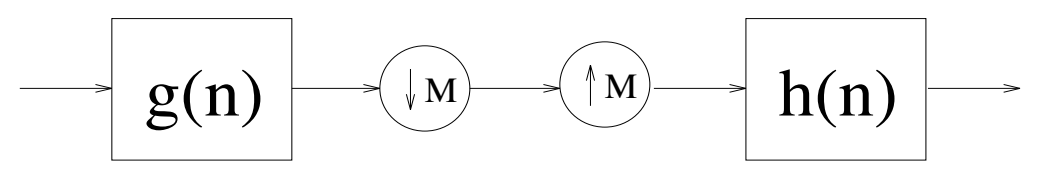

Figure 1: The multirate implementation of a filter.

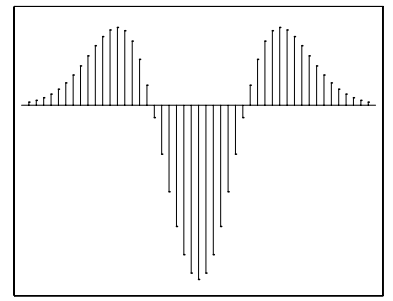

(a)

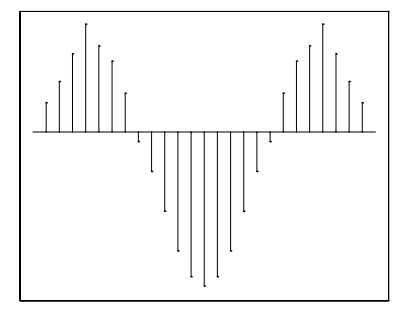

(b)

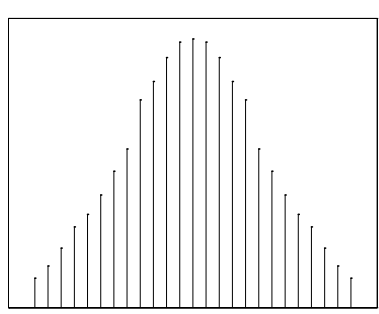

(c)

Figure 2: The kernel $d(n)$ to be approximated (a), and the filters $g(n)$ (b) and $h(n)(\mathrm{c})$ minimizing $\mathcal{E}^{2}$. 